\title{
Structural Analysis and Matching of Shape by Logical Property*
}

\author{
Nanhyo Bang and Kyhyun Um \\ Department of Computer and Multimedia Engineering, Dongguk University \\ Pil-dong 3 Ga 26, Chung-gu, Seoul, 100-715, Korea \\ $\{j$ jang, khum\}@dgu.ac.kr
}

\begin{abstract}
The shape is one of most important feature for characterising an object. However, most shapes that are expressed with primitive uniform features have difficulty reflecting their logical and structural properties. In this paper, we propose a structural analysis scheme for the shape feature structured by logical properties, as well as a similar retrieval method. A shape is represented as a set of curve segments with a specific pattern. As a fundamental unit, a curve segment has adaptive features based on the logical property of its pattern. The relationship information of curve segments is expressed as a structural feature. We also use it as a feature for "coarse-fine" matching because our shape features have global characteristics as a structural feature and local characteristics as an adaptive feature of shape. Our experiments show that structuraladaptive features through logical analysis result in effectively classifying shapes according to their cognitive characteristics. Various experiments show that our approach reduces computational complexity and retrieval cost.
\end{abstract}

\section{Introduction}

Many information retrieval systems use images are faced with the challenge of representing and retrieving images. These systems have domain-dependent features in terms of retrieval or show improper results. This is because most researches have represented image as a primitive feature. Most primitive features express image as a low-level feature and numerically define the correlation between points. Researchers who have studied content-based retrieval systems define image as data with rich content. They classify features that compose image into a primitive feature and a logical feature[1][2]. A logical feature connotes a complex content, including semantic information. In [3], content is defined as "an understanding of the semantics of objects." Therefore, features that represent rich contents of an image must have a logical property in order to properly reflect semantic contents. In this aspect, the shape feature among many features that represent image has been widely used by virtue of its ability to recognize similar images.

This research has been supported by Korean Science and Engineering Foundation No. R012002-000-00298-0 
In this paper, we analyze shape according to the composition of its structural unit with logical properties. Fundamental units with adaptive features and structural relations between these units represent the logical contents of shape. We use a curve segment as a fundamental element to express the appearance of a shape. Structural and adaptive features are extracted through a logical and structural analysis using a curve segment. The structural feature as a global feature is used as a coarse-matching feature because it contains pattern and relationship information of curve segments comprising the whole shape. The adaptive feature, on the other hand, is a local feature. Using this feature produces correct results because of its ability to reflect specific geometrical properties of each pattern segment. Our experiments show how retrieval cost is reduced when conducting a search using these structural-adaptive features.

This paper is organized as follows: In chapter 2, we will explain a logical analysis scheme of the shape. In chapter 3, we will explain extraction methods of structural and adaptive features. A matching process and similarity function is explained in chapter 4. Experiment results and performance are shown in chapter 5. Related works are summarized in chapter 6 , and the conclusion is drawn in chapter 7.

\section{Structural Analysis of Shape}

We define a shape as a set of curve segments with a pattern. Through preprocessing, we extract the necessary primitive feature in order to define the logical property and fundamental unit. Using this, we structure shape.

\section{Preprocessing}

We extract contour points from image's object to represent shape. However, because the size of contour points is too large, we use an adequate number of interest points that reflect a characteristic of a shape that is extracted from contour points. Interest points are aligned clockwise and described as a set $S=\left\{p_{1}, p_{2}, \ldots ., p_{n}\right\}$, where an interest point is denoted by $p_{i}$ and subscript $n$ is the index number of the aligned interest point. A $p_{c}$ is the gravity point of a shape. Two features are extracted from these interest points: $\theta$ and $\alpha$ feature.

- $f \theta\left(p_{i}\right)=\theta_{i}=\angle p_{1} p_{c} p_{k}, 1<k \leq \mathrm{n}$

- $\quad f I A\left(p_{i}\right)=\alpha_{i}=\angle p_{i-1} p_{i} p_{i+1}, 0<\alpha_{i}<360$

In our work, a curve is separated using $\alpha$ feature of interest points that comprises a concave part. The separated segment is defined as curve segment, $C S=\left\{p_{i} \mid i s \leq i \leq i e, \quad f I A\left(p_{i s}\right), f I A\left(p_{i e}\right)>180\right\}$

If CS includes two interest points, it becomes a concave segment. CS merges with the next CS into the new CS, if the next CS is also a concave segment. CS is a fundamental unit for the structured analysis of a shape. 


\section{Structure of Shape}

Curve segments have specific patterns according to logical property. Logical properties are characteristics that logically define the curve pattern of CS. We redefine CS with logical properties as PS(Pattern Segment), and we generate PSS(Pattern Segment Sequence) as our shape feature to represent the structure and characteristic of shape. The logical properties of CS are expressed as adaptive features. The relationship between them is defined as follows:

- $\quad$ Shape $\mathrm{S}=\mathrm{PSS}$

- $\operatorname{PSS}=\{\mathrm{PS}\}+$ structural features

- $\mathrm{PS}=\mathrm{CS}+$ pattern type (with adaptive features that reflect logical properties by pattern type)

A CS has two logical properties: convex/concave property and turning property.

Definition 1. Convex/Concave Property. If interest points included in CS are all concave interest points or convex interest points, we define this CS with convex/concave property. Therefore, it is $\forall \operatorname{fIA}\left(p_{k}\right)>180$ or $\forall f I A\left(p_{k}\right)<180$, when $p_{k} \in C S$ and $i s<k<i e$.

Turning property means that CS changes direction or goes around a specific center.

Definition 2. Turning Property. Let CS be a sequence of two CS: $C S_{1}=$ $\left\{p_{i s}, \ldots, p_{m}\right\}, C S_{2}=\left\{p_{m}, \ldots p_{i e}\right\}$, where $p_{m} \in C S$. One of them is CS with conver property and the other is CS with concave property. We define this CS as one with turning property if MBR(Minimal Boundary Rectangle) generated from convex CS overlap with MBR generated from concave CS.

PS is CS that has a specific pattern. First, CS is classified into either a concave curve segment or convex curve segment according to definition 1 . Then, a turning curve segment is classified according to definition 2. The PSS is thus defined as $P S S=\left\{P S_{1} \mid P S_{1}=<p t\right.$,sflistaflis $\left.\left.\triangleright, \quad 1 \leq i \leq s\right\} \quad 1 \leq i \leq s\right\}$ where, $p t$ is a pattern type, sflist is structural featuress, and aflist represents adaptive features of PS. PSS is stored in a database where information of interest points are saved.

\section{Features Extraction of Pattern Segment}

PSS has structural and adaptive features. PS that is an element of PSS shows a geometrical property of the partial curve to compose a shape through adaptive features.

\section{Structural Features}

Related information between PSs is used in order to compare the whole structure of shape. The $\alpha$ feature of interest point that divides adjacent PSs is simply used as a structural feature in our work. However, another shape is made by case even if they have the same $\alpha$ feature. The (a)(b) of Figure 1 shows such an example. 


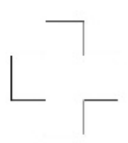

(a)

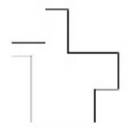

(b)

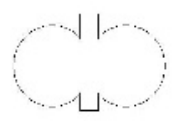

(c)

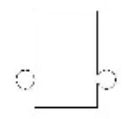

(d)

Fig. 1. (a) original shape, (b) an inner angle is the same as (a), but is a different shape. (c) original shape, (d) appearance of the curve is the same (c), but has a different scale ratio.

When a set of curves is compared, its structural feature will be reflected because the difference in the shape depends on the size difference even if the adjacent curves are similar to each other (Fig.1 (c)(d)). As explained above, when the pattern type of PSS of two shapes being compared is the same one, its structural features are used to calculate the structural difference of the shape.

- $\alpha$ feature of a PS's last interest point, adist - Because this feature is most simple feature to represent relationship between adjacent PSs, we use it when measuring structural similarity.

- Pattern range, pr -The start base angle ( $s a$ ) and end base angle ( ea ) features are appended in order to solve the problem that $\alpha$ feature of two shapes is the same but appear to be different (Fig.1 (a)(b)). The start base angle is $\angle p_{i s+1} p_{i s} p_{i e}$ and the end base angle is $\angle p_{i e-1} p_{i e} p_{i s}$.

- Size ratio of PS, sr - To solve problems such as (c) and (d) of Fig. 1, we extract the proportion of a PS to whole shape.

\section{Adaptive Features}

Adaptive features are features that reflect the logical and geometrical properties of each PS. They are called "adaptive features" because each of the different features adequately reflects the property of PS and is used in each of them. Representative adaptive features are as follows:

- Average of $\alpha$ feature, cc $\alpha$ - This feature represents the degree of circularity.

- Eccentricity $e$ - This represents the eccentricity of the polygonal segment.

$$
\begin{aligned}
& \text { lleng }=\overline{p_{m m} p_{m d}}, \quad p_{m m}=\left(p_{i s}+p_{i e}\right) / 2, p_{m d}=\operatorname{Max}\left(\underset{k=i s+1}{\forall} d p\left(p_{i s} p_{i e}, p_{k}\right)\right) \\
& \text { sleng }=\operatorname{Max}\left(\underset{s i=i s}{\forall} \operatorname{\forall d-1} d p\left(p_{m m} p_{m d}, p_{s i}\right)\right)+\operatorname{Max}\left(\underset{e i=m d+1}{\forall} d p\left(p_{m m}^{i e} p_{m d}, p_{e i}\right)\right) \\
& e=\text { sleng } /(\text { sleng }+ \text { lleng })
\end{aligned}
$$

A function $d p(L, p)$ return the length of perpendicular line from point $p$ to line $L$. If $e=0.5$, the MBR of the segment is almost a regular polygon. If $e>0.5$, it will appear as an elongated segment pointing right to left. Otherwise, it will appear as an elongated segment pointing upwards.

- Turning angle ta - A ta is calculated from PS with partial or global turning properties. This feature extracts MBR from the concave segment of PS with a turning property. The center points of turn and turning angle are extracted from this MBR. 


\section{Matching}

Similarity retrieval is executed using PSS. First of all, we filtered shapes that are most similar according to the structural information of PSS in order to reduce search cost. Similarity costs of structural and adaptive features are combined to calculate an integrated similarity cost.

\section{Pattern Segment Matrix}

For structural matching, the PSS feature of shape D in the database is compared with query shape Q. A Pattern Segment Matrix (PSM) is generated in order to find a comparably similar segment sequence. PSM expresses a value to represent the identity of pattern types between the PSSs of two shapes as elements of the matrix:

$$
M_{r c} \text { 's value }=\left\{\begin{array}{ll}
1, & Q P S_{r} \cdot p t=D P S_{c} \cdot p t \\
0, & Q P S_{r} \cdot p t \neq D P S_{c} \cdot p t
\end{array} \quad\left(Q P S_{r} \in Q P S S, D P S_{c} \in D P S S\right)\right.
$$

PSS of Q (QPSS) and PSS of D (DPSS) are used to represent the row and column axis of the matrix, M. After generating the matrix, we test whether the value of 1 continuously appears in a clockwise direction if M's value is 1 . Again, we test the PSM made with QPSS, which was generated in reverse to find the symmetric shape. CSP(Candidate Similar Pattern sequence) are sequential segments with a similar pattern type between Q and D.CSP represent PSs with similar pattern in a diagonal direction in PSM. The CSP is as $C S P=\left\{C S P_{i} \mid C S P_{i}=<s q\right.$, sd, sleng $\left.>\right\}$ where, $s q$ stands for index $r$ of QPSS, the starting point of sequence, and $s d$ is $c$ index of DPSS, and sleng is length of the sequence.

\section{Similarity Cost}

The $r C S P$, the segment sequence most similar to the shape of query and shape of database, is selected from PSM. The $r C S P$ is a CSP that is selected due to structural matching.

$$
r C S P=\operatorname{MAX}\left(\left(\alpha d i s t_{i}+p r_{i}+s r_{i}\right) / 3\right), \quad\left(\alpha d i s t_{i}, p r_{i}, s r_{i} \in \forall C S P_{i}\right)
$$

Among the CSP, we select $r C S P$ with a maximum similarity value between structural features. The adaptive features of two PSs included in $r C S P$ are compared. PtDist is the distance between adaptive features of two PSs. A similarity of adaptive features extracted according to segment pattern is calculated using Euclidian distance. The total similarity cost is defined as

$$
\begin{aligned}
\text { SimCost }= & \frac{(Q r+D r)}{2} \times \text { Dist }(\text { Structural features }) \\
& \times \text { Dist }(\text { Adaptivefeatures })
\end{aligned}
$$

In (3), if value of Dist (Structural features) exceeds 0.8, then its value is 1 . The $Q r$ and $D r$ are the ratios of PSs belonging to $r C S P$ in the whole shape. 


\section{Experiments}

The feasibility of structured analysis based on the logical characteristic of a shape and the utility of its adaptive features can be verified through experiments. For these, we use two test databases: 1,100 shapes from SQUID and 216 shapes selected from MPEG-7 test database. A search is then carried out with QBE.

\subsection{Experimental Setup and Results}

We have developed a prototype system with MS Visual $\mathrm{C}++6.0$, and Oracle 8i. Figure 2 shows a block diagram of our prototype system.

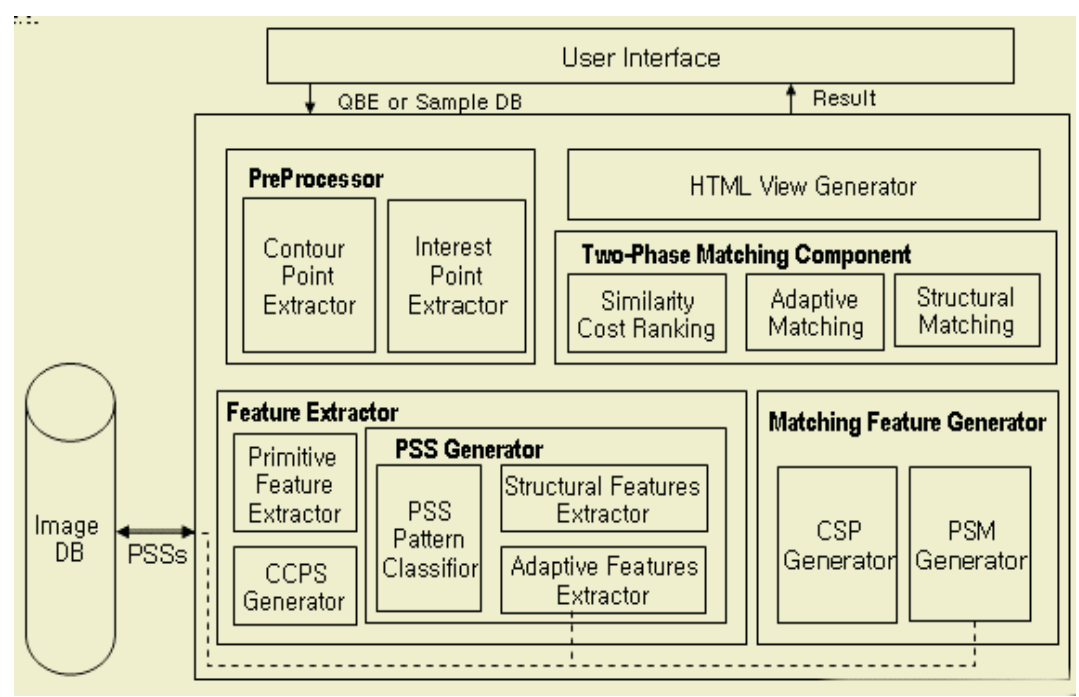

Fig. 2. Block Diagram of our Prototype System.

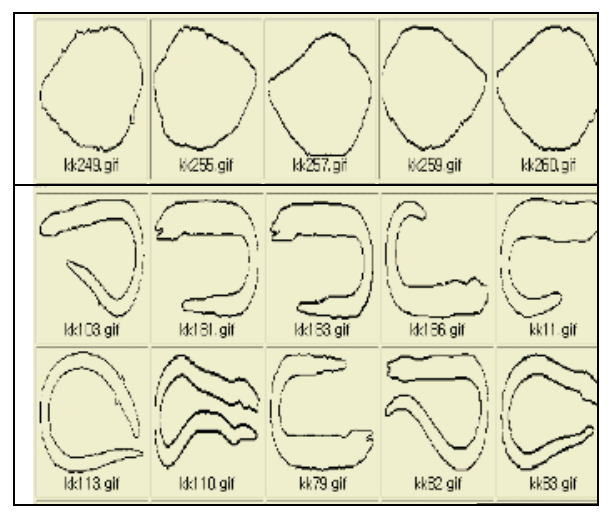

Fig. 3. Classified Shapes.

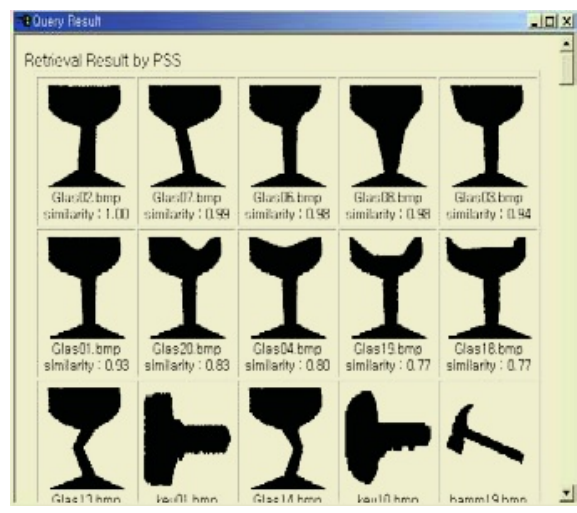

Fig. 4. Query Results. 
Figure 3 shows a retrieval result using only the PSS and logical properties from SQUID data. The global turning feature of the shapes in the second row of Figure 3 is applied. This retrieval result is the same as results produced by humans. Figure 4 is a result from the second test database, which has similar candidate shapes of partially various transformations that are highly similar. On an average, the extracted features are as follows: For SQUID, contour point is 693, interest points are 22 and PS is 8 . For the second database, contour point is 878 , interest points are 23 and PS is 7 .

\subsection{Performances}

We complete a performance evaluation of shape features with features that are used, such as storage cost and feature extraction cost. We then compare our scheme with some representative methods such as the Grid Based Method and the Fourier Descriptors Method in [4].

Table 1. Storage Cost and Feature Extraction Cost Comparison.

\begin{tabular}{|c|c|c|c|}
\hline Criteria & Grid Based Method & $\begin{array}{c}\text { Fourier Descriptors } \\
\text { Method }\end{array}$ & Ours \\
\hline $\begin{array}{l}\text { Storage } \\
\text { cost }\end{array}$ & $2 *((\mathrm{mj} / \mathrm{gn}) 2) / 8+8$ & $8 * \mathrm{fn}$ & $13 * \mathrm{~m}$ \\
\hline $\begin{array}{l}\text { Extraction } \\
\text { cost }\end{array}$ & $\begin{array}{l}\text { To find the major axis } \\
\text { O(N2) } \\
\text { To find the minor axis } \\
\text { O(N) } \\
\text { To rotate and scale } \mathrm{O}(\mathrm{N}) \\
\text { To generate a binary num- } \\
\text { ber } \mathrm{O}(\mathrm{N} 3)\end{array}$ & $\begin{array}{l}\text { To find the centroid } \\
\text { O(NlogN) } \\
\text { To find all radii and } \\
\text { compute FD coeffi- } \\
\text { cients } \mathrm{O}(\mathrm{r} 2) \\
\text { To generate signature } \\
\mathrm{O}(\mathrm{r})\end{array}$ & $\begin{array}{l}\text { To extract the inter- } \\
\text { est point } \mathrm{O}(\mathrm{NlogN}) \\
\text { To segment PSS } \\
\mathrm{O}(\mathrm{m}) \\
\text { To extract struc- } \\
\text { tural-adaptive fea- } \\
\text { tures } \mathrm{O}(\mathrm{m})\end{array}$ \\
\hline
\end{tabular}

In Table $1, m j$ is the major axis size and $g n$ is the grid cell size for the grid-based method. The $f n$ is the number of Fourier coefficients and $r$ is the number of radii for the Fourier Descriptors Method. In our scheme, $m$ is the number of interest points. Our scheme shows better performance from the point of view of feature extraction cost. This is almost the same as the generation time of TPVAS in [4]. Tables 2 and 3 show computation cost to prepare internal data for searching and the space requirement to use in each searching step.

Table 2. Computation Cost.

\begin{tabular}{|l|l|}
\hline Step & Cost \\
\hline $\begin{array}{l}\text { To make Pattern } \\
\text { Matrix }\end{array}$ & $\mathrm{O}\left(\mathrm{s}^{2}\right)$ \\
\hline To make CSPs & $\mathrm{O}\left(\mathrm{s}^{2}\right)$ \\
\hline To generate rCSP & $\mathrm{O}(\mathrm{cs})$ \\
\hline
\end{tabular}

Table 3. Used Features and Size.

\begin{tabular}{|l|l|}
\hline Search step & Used Features and Size \\
\hline Structural & $\begin{array}{l}\text { Pattern type feature }: 6 \text { bit } * \\
\text { s } \\
\text { Structural feature }: 26 \text { bit } * \text { s }\end{array}$ \\
\hline Adaptive & $\begin{array}{l}\text { Adaptive features }: \text { average } 9 \\
\text { byte } * \text { s }\end{array}$ \\
\hline
\end{tabular}


In Tables 2 and 3, $s$ is the number of a curve, and $c s$ is the number of CSP. On the average, $s$ is such a small number that it is about $1 / 3$ of interest points. Our scheme has a merit to search with only a small quantity of structural features. We analyzed how search cost can be decreased on several representative query set for the second database.

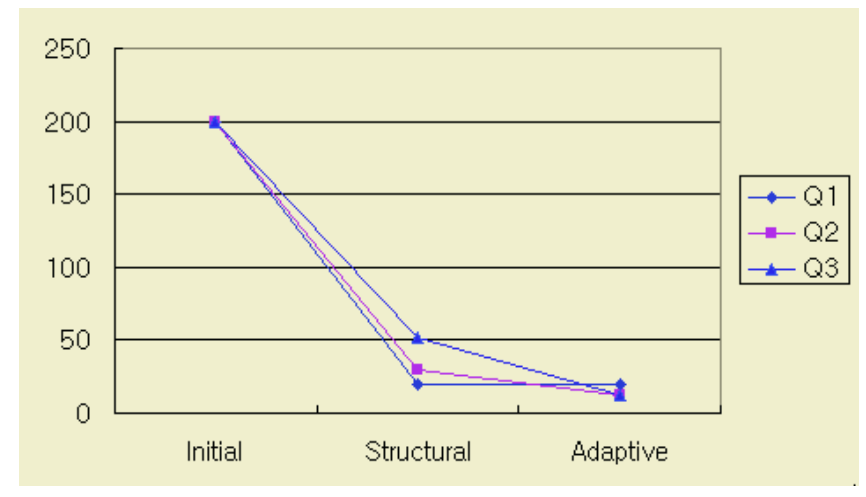

Fig. 5. Search Space Comparison Graph.

Query set Q1 is queries that use shape data, which are then transformed by rotation, symmetry and scaling to the same shape. Query set Q2 is queries that use shapes with 2 or 3 PSs. Query set Q3 is queries that use shapes with more than 7 PSs. We then sort the data results according to similarity in value. The digit numbers on the $y-$ axis refer to the number of images. Q1 approximates results with only structural features. In Q2, with a few PSs, shapes searching all appear as a result of the comparatively high similarity value. However, in Q3, there are more objects to search. This is because the partial matching value is included in the high ranking of results while some parts of PSs, with a query match with shapes, have fewer PSs.

\section{Related Works}

Contour- and region-based features are part of the representation method (Curvature Scale Space and Zernike Moment) of shape information adopted by the MPEG-7. Main topics covered by contour-based researches include matching strategies with contour points or additional information such as curvature of shape as well as methods for interest point extraction that preserves the original shape contour while reducing dimensionality [5]. In [6], Curvature Scale Space (CSS) information is taken at multi-resolution. In [7], e-envelope characteristics are represented by contour points. In [8], an object's shape is presented with line segments, and features derived from the line segments are used for matching. Using contour points, the multi-level resolution pyramid structure is designed and used for retrieval [9]. As an effort to solve these problems, concave/convex segment is extracted from the contour as shown in [10], and similarity search is conducted using dynamic programming. However, poor search performance is a common problem among researches that use these primitive features. 


\section{Conclusion}

In this paper, we suggested methods to analyze a shape logically and to structure designed properties. A shape is composed of a curve segment as a fundamental unit. A curve segment has specific logical properties according to geometrical pattern. Through this, pattern segment is generated from curve segment. Pattern segment sequence possesses structural features between adjacent pattern segments. We use adaptive features extracted according to the segment's geometrical features. It is possible to increase the accuracy of search in comparison with ordinary point features. Since pattern segment reflects local features, it can be used for searching partial or overlapped objects. Experimental results show that our shape feature adequately represents object shape; and that it is possible for the retrieval method to improve accuracy and efficiency. Further researches for the proper classification of PS's pattern are needed so that it can represent the semantic feature of shape and enhance search performance. The designed logical properties are applied to various shapes of closed polygons. These logical features extend meaningful semantic features by integrating with other features.

\section{References}

1. Venkat N. Gudivada and Vijay V. Raghavan, "Content-Based Image Retrieval Systems," IEEE Computer, September 1995, pp. 18-22.

2. C. H. C. Leung and Z. J. Zheng, "Image Data Modeling for Efficient Content Indexing," Proc. Intl. Workshop on Multi-Media Database Management Systems, August 28-30, 1995, pp. 143-150.

3. A. Desai Narasimhalu, "Special Section on Content-based Retrieval," ACM Multimedia Systems, No. 3, 1995, pp.1-2.

4. Maytham Safar, Cyrus Shahabi and Xiaoming Sun, "Image Retrieval By Shape: A Comparative Study," IEEE Intl. Conference on Multimedia and Expo (I),2000, pp.141-154

5. D. Geiger, T. L. Liu and R. V. Kohn, "Representation and Self-similarity of Shapes," IEEE Transactions on Pattern Analysis and Machine Intelligence, Vol. 25, Issue No. 1, 2003, pp. 86-99.

6. F. Mokhtarian, "Silhouette-based Isolated Object Recognition through Curvature Scale Space," IEEE Transactions on Pattern Analysis and Machine Intelligence, Vol.17, 1995, pp. 539-544.

7. I. Fudos, L. Palios and E. Pitoura, "Geometric-similarity Retrieval in Large Image Bases," Proc. of 18th International Conference on Data Engineering, 2002, pp. 441-450.

8. P. N. Suganthan, "Shape Indexing Using Self-organizing Maps," IEEE Transactions on Neural Networks, Vol. 13, Issue No. 4, July 2002, pp. 835-840.

9. M. Mignotte, "A new and simple shape descriptor based on a non-parametric multi-scale model," Proc. of. International Conference on Image Processing, Vol. 1, 2002 , pp. 445448.

10. E. Milios and E.G.M. Petrakis, "Shape Retrieval Based on Dynamic Programming," Trans. Image Proc., Vol. 9, no. 1, 2000, pp. 141-146 